\title{
Historia del Departamento de Obstetricia y Ginecología del Hospital Universitario de La Samaritana
}

El Hospital Universitario de La Samaritana fue construido en un lote de 14.680 varas cuadradas, ubicado en el barrio las Cruces (Cra. $8^{a}$ Calle $1^{\text {a }}$ ), el cual fue vendido por los hermanos Teodiceldo y Miguel Macías a la Gobernación de Cundinamarca, mediante escritura pública No. 1817 de la Notaría Cuarta, con fecha 25 de agosto de 1933, siendo Gobernador del Departamento de Cundinamarca el Doctor Liborio Cuéllar Durán y Secretario de Gobierno el Doctor Carlos Suárez Latorre.

E1 26 de octubre de 1933, bajo la administración de Alfredo Zerda y posteriormente de Reinaldo Frasser, se iniciaron las obras de construcción, actuando como Arquitecto Carlos J. Lascano y como Ingeniero Francisco Cano.

Al tiempo que se iban adelantando las obras, se iniciaron las actividades científicas encaminadas a la lucha antivenérea, ya que en el sector de las Cruces se ubicaban las más importantes fábricas de chicha (las famosas chicherías), lo cual conllevaba a un gran número de casas de lenocinio en el sector. El nombre de La Samaritana nace entonces como un símbolo de apoyo a las trabajadoras sexuales, quienes fueron las primeras beneficiarias de las labores de la institución. La atención antivénerea fue responsabilidad del Doctor Jorge Cavelier, distinguido urólogo, quien fue el impulsor y gestor del hospital hasta la década del 70, habiendo ocupado altos cargos entre ellos el Ministerio de Higiene y Salud Pública; la sección ginecológica fue encomendada al Doctor Humberto H. Correa Sierra, quien fue el creador del departamento, siendo jefe hasta el año 1984. Es importante recordar al Profesor Correa como una gloria de la medicina colombiana, alto, elegante, con un fino humor bogotano, matizado con cierta influencia francesa, producto de sus años de estudio en París. De recia personalidad y gran carácter, mucho le debe la ginecología colombiana, y más aún, los que fuimos sus discípulos, a este insigne galeno, poseedor no sólo de una especial habilidad quirúrgica, de una memoria prodigiosa y un espíritu docente vivaz y ameno, sino también de una distinción que le permitía ejercer sus actividades con una altura espontánea y natural.

Los siguientes fueron los tratamientos utilizados en los años 30 y 40 :

- Chancros: Tópico García, tópico amarillo y pomada resolutiva

- Blenorragia: Mercurio cromo, sulfalinamida, galvanocauterizaciones, glicerina ictiolada, ácido láctico, nitrato de plata, devegan y glicerina ferricada.

- Vegetaciones: Nitrato de plata, pomada al calomel, ácido acético.

- Linfogranuloma venéreo: Tártaro emético y fuadina.

- Sarna: Pomada Hardy

- Cistitis: Instilaciones de nitrato de plata

En la década de los 40 los sueldos mensuales en el hospital eran del siguiente orden:
- Médico permanente 180 pesos.

- Médico practicante 60 pesos.

- Jefe del laboratorio 120 pesos.

- Enfermera 30 pesos.

Por esta época la consulta externa de gineco-obstetricia de Bogotá se repartía de la siguiente manera:

\section{Consulta Ginecológica}

- Cruz Roja Nacional: Dr. Zoilo Cuéllar Durán.

- Hospital San Juan de Dios: Dr. Guillermo Araos Frasser.

- Hospital San José: Dr. Manuel Antonio Rueda.

- Instituto de Higiene Social: Hospital de La Samaritana.

\section{Consulta Antivenérea}

- Cruz Roja Nacional: Dr. Manuel José Silva.

- Hospital San Juan de Dios: Dr. Gonzalo Reyes García.

- Instituto de Higiene Social: Hospital de La Samaritana.

\section{Consulta Prenatal}

- Centro: Dres. Luis Urdaneta y Carlos Gómez

- Cruz Roja Nacional: Dr. Pinto Valderrama

- Chapinero: Dr. Carlos Navarro Tovar

- Hospital San Juan de Dios: Dr. Nicolás Torres Barreto

El Hospital gradualmente entró en un proceso de generalización de la atención médica y ya promediando la década de los 50 el Departamento inició la atención obstétrica y se convirtió en una institución pionera en la cirugía ginecológica gracias a la labor del Dr. Roberto Vergara Támara, distinguido galeno e hidalgo caballero de la medicina.

En el año de 1957 ingresó al hospital como médico adscrito al Departamento el Dr. Joaquín Luna Uribe, quien acababa de terminar su residencia en el Hospital San José y, al lado del Dr. Vergara Támara, comenzó una carrera como brillante ginecólogo y maestro de varias generaciones de especialistas, labor que en la actualidad continúa desarrollando. No hay calificativos para describir la calidad humana y profesional del profesor Joaquín Luna Uribe.

Creo no equivocarme al distinguirlo como uno de los mejores cirujanos ginecólogos que existe en el país. Innovador en la técnica quirúrgica, tranquilo y diestro ante la complicación intraoperatoria, dominador en el manejo del instrumental y, ante todo, docente generoso y cálido. Poseedor de una personalidad sencilla y jovial, hábil pescador que trabaja con la caña de pescar con la misma destreza que con la tijera de tejidos, el Dr. Luna, hoy en día profesor emérito y honorario del Departamento, ocupa en el corazón de todos sus discípulos el lugar de un padre, de un maestro y de un amigo.

En el año de 1978 se inició el programa de postgrado con el aval de la Universidad Javeriana, institución vinculada al hospital prácticamente desde sus inicios. Además de los profesores ya mencionados, han sido decentes del Departamento los siguientes especialistas: 
- Manuel Aristizábal

- Cecilia Espinosa de Delgadillo

- Luis Maldonado

- Luis A. Giraldo

- Jaime Martínez

- Doris Blandón

- Diego Gutiérrez

- Margarita Hurtado

- Elías Castel

- Mauricio Mendieta A.

- David Eissen

- Byron Cardoso

- Joaquín Gustavo Luna

- Javier Bonilla

- Camilo Libos S.

El profesor Humberto H. Correa S. ejerció la jefatura del Departamento hasta el año de 1984, cuando fue remplazado por el Dr. Luis Ernesto Pontón, quien a su vez dejó el cargo en manos del Dr. Joaquín Luna Uribe en el año de 1990 al ser nombrado director del hospital. El Dr. Luna ocupó la jefatura hasta mayo de 1994 cuando fue remplazado por el Dr. Luis Fernando Castaño, quien a su vez ejerció el cargo hasta mayo de 1995, fecha en que el Dr. David Vásquez Awad asumió la dirección del Departamento.

El Departamento de Obstetricia y Ginecología cuenta actualmente con todos los servicios requeridos como son la clínica de menopausia, clínica de alto riesgo obstétrico, clínica de infertilidad y endocrinología ginecológica, clínica de oncología y clínica de incontinencia urinaria. Igualmente se realizan procedimientos de alta tecnología con equipos nuevos de colposcopia, histeroscopia, radio cirugía, video laparoscopia operatoria, mamografía, ecocardiografía fetal, y de esta manera desde el punto de vista asistencial y docente el Departamento se coloca a la vanguardia, situación reflejada en el último Congreso Nacional de Ginecología y Obstetricia (Medellín 1996), cuando se recibió el premio al mejor trabajo científico realizado por un residente.

El cuerpo médico del Departamento es el siguiente:

- Profesor emérito y honorario:

Dr. Joaquín Luna Uribe

- Jefe de Departamento:

Dr. David Vásquez Awad

- Instructores del Departamento:

Dr. Diego Cabrales de la Pava

Dr. Luis Fernando Castaño Molano

Dr. Mauricio Durán Zamudio

Dr. Imac de María Guerrero Rodríguez

Dr. Eduardo Naranjo Mejía

Dr. Manuel Antonio Plata García

- Instructores Ad-honorem

Dr. Byron Cardoso Medina

Dr. Juan Carlos Vargas Rugeles

- Residentes Dr. Alberto Angulo Rodríguez

Dr. Oscar Mauricio Caina Bernal

Dr. Luis Antonio Juagibioy Jamioy
Dr. José Roberto Gómez Duque

Dr. Ricardo Montoya González

Dr. Mauricio Sarmiento Sarmiento

Dr. Ernesto Forero Villamizar

Dr. Guillermo Obando Díaz

Dr. Juan Carlos Romero Contreras

Dr. Luis Alfonso Vallejo Arcila

Del Departamento han egresado los siguientes especialistas en orden croniológico:

- Jorge Linero

- Gilberto Martínez Morales

- Marco Espinosa

- Luis Fernando Castaño

- Nelly Camacho

- Severo Pérez

- Doris Blandón Rodríguez

- Jairo Prada

- Jorge Luis Becerra

- Benjamín Navarro

- Ramiro Roa

- Dora María Suárez

- Jairo Linares

- Miguel Darío Rico

- Hermes Díaz

- Humberto Rodríguez

- David Vásquez Awad

- Elías Castel Amon

- Carlos Eduardo Gómez Franco

- Mauricio Mendieta Albornoz

- Rafael Hinestrosa Ayala

- Luis Eduardo Castellanos G.

- Alonso Perdomo Villa

- Manuel Antonio Plata García

- Jorge Enrique Córdoba Villota

- Javier Eduardo Bonilla Amaya

- Byron Cardoso Medina

- Adriana Soto Montoya

- Jorge Enrique Londoño Rodríguez

- Luz Francy Torres Díaz

- Diego Bayardo Cabrales de la Pava

- Fabio Ernesto Quijano García

- Mauricio Durán Zamudio

- Pablo Vargas Poveda

- Imac de María Guerrero Rodríguez

- Diana Solórzano Garavito

- Camilo Libos Sayegh

- Camilo Mojica Rivadeneira

- Nelson Ricardo Rincón Molano

- Edgar Sánchez Caicedo

- Luz Jalime Arbeláez Correa

- Germán Hernández Cely

- Guillermo Navarro Naranjo

DAVID VASQUEZ AWAD, M.D.

Jefe Departamento Obstetricia y Ginecología Miembro Junta Directiva Hospital de La Samaritana 